\title{
DIE ALBANISCHEN TANZLIEDER IN BYRON'S CHILDE HAROLD.
}

Lord Byron hat in der anmerkung zur 32. stanze des zweiten gesanges seines Childe Harold einige proben albanischer volkspoesie mitgeteilt, die er in der umgegend von Athen, wo bekanntlich noch hente die landbevölkernng fast ausschliesslich albanisch ist, durch einen des schreibens kundigen hat aufzeichnen lassen. Er bemerkt dazu: 'As a specimen of the Albanian or Arnaout dialect of the Illyric, I here insert two of their most popular choral songs, which are generally chanted in dancing by men or women indiscriminately...... It is to be observed, that the Arnaout is not a written language: the words of this song, therefore, as well as the one which follows, are spelt according to their pronunciation. They are copied by one who speeks and understands the dialect perfectly, and who is a native of Athens'. Ein versuch, diese kleinen strophen herzustellen und zu verstehen, ist meines wissens nur einmal gemacht worden, nämlich von Xylander in seinem buche 'Die Sprache der Albanesen oder Schkipetaren' (Frankfurt a. M. 1835) s. $145 \mathrm{ff}$. Da aber Xylander das Albanische wesentlich nur aus einer ubersetzung des Neuen Testamentes kannte, ist er nicht gelungen. Nur ganz wenige worte hat er richtig verstanden, einige falsch, die meisten gar nicht.

Ich finde noch an einem andern orte eine erwähnung der Byron'schen texte. Ludwig Steub erzählt in seinen 'Bildern aus Griechenland' (Leipzig 1841), I 160 bei der schilderung seines besuches auf der von Albanesen bewohnten insel Hydra: 'Es erregte ein freudiges erstaunen, als ich einst in einer gesellschaft von Hydräern die dem zweiten gesange von Childe

Anglia. N. F. III. 
Harold angehängten albanesischen lieder vorwies. Man ging gleich darlber, sie zu lesen und zu erklären, fand aber, wie herr hauptmann von Xylander, dass sie entweder unrichtig niedergeschrieben, oder durch druckfehler entstellt sein misssen, denn unter allen diesen distichen war kein einziges, das ungezwungen einen annehmbaren sinn gegeben hätte'. Diese tatsache macht dem scharfsinne der trefflichen bewohner von Hydra keine sonderliche ehre. Denn, wie sich gleich herausstellen wird, die lieder sind durchaus nicht so fehlerhaft aufgezeichnet, dass nicht ein der sprache kundiger beim lauten vorlesen wenigstens zum teil sie hätte verstehen können.

$\mathrm{Zu}$ dem hier mitgeteilten versuche einer herstellung und ubersetzung dieser textproben bin ich zunächst durch Doctor Nerutsos-Bey in Ramleh bei Alexandrien veranlasst worden, einen aus Griechenland geburtigen Albanesen, der mir vor längerer zeit bereits seine lesung dieser zweizeiler, mit ausschluss der letzten, mitteilte. Ich habe sie mit vielem vorteil benutzt, wenn ich auch an einigen stellen von ihr abzuweichen genötigt war. Die liedchen gehören sämtlich der gattung kleiner, zweizeiliger improvisationen an, ron denen Dr. Reinhold in seinen 'Noctes pelasgicae' (Athen 1855) eine ganze menge mitgeteilt hat. Auffallender weise findet sich grade von den Byron'schen liedern dort keines; denn wenn auch diese aus der umgegend von Athen, die Reinhold's von den inseln stammen, so weiss man doch, wie diese zweizeiler sonst auch ubers meer wandern. Ich selbst habe in den dörfern Attikas mehrere solcher distichen aufgezeichnet, unter denen sich ebenfalls keines der Byron'schen findet. Byron hat die siebzehn distichen als strophen zweier grösserer lieder betrachtet, von denen das erste zehn, das andere sieben enthält. Es ist ihm nicht entgangen, dass die beiden letzten sich in ihrer metrischen form von den ubrigen unterscheiden: ' $I$ believe the two last stanzas, as they are in a different measure, ought to belong to another ballad'. In wirklichkeit handelt es sich hier iberhaupt um keine 'ballads'; die zweizeiler werden beim tanz an einander gereiht, ohne dass der inhalt der einzelnen ihre verknupfung immer sonderlich rechtfertigt. Das metrum der ersten funfzehn ist eine trochäische tetrapodie, entweder vollständig (6. 8. 9.11.12.13.14.15?) oder mit unterdruckung der letzten senkung (1.2.3.4.5.7.10); die beiden letzten zeigen 
das mass, das unter dem namen des politischen verses in der neugriechischen volksdichtung weit verbreitet ist, den funfzehnsilbigen, nach der achten silbe mit einer cäsur versehenen jambischen vers, Es ist kein zweifel, dass hier lediglich eine nachahmung griechischer volksdichtung vorliegt; auch die trochäischen dimeter werden in den griechischen zweizeiligen improvisationen verwendet, wenn auch weniger bäufig als der politische vers, und sind der echten, nationalen albanischen volkspoesie fremd. Aehnlich bedienen sich die Albanesen Suditaliens der kunstrollen formen italienischen volksgesanges fur ihre lieder.

Ich stelle im folgenden die bei Byron vorliegende uberlieferung und meine herstellung einander gegentiber, und ebenso die Byron'sche übersetzung der meinigen. Fur die schreibung des Albanesischen verweise ich auf meine 'Albanesische Grammatik' (Leipzig 1888) § 1. Den zweizeilen habe ich, abweichend von Byron, fortlaufende nummern gegeben.

1.

$B o, B o, B o, B o, B o, B o$,

Nuciarura, popuso.

Lo, Lo, I come, I come; be thou silent.
Bo bo bo, bo bo bo, na tš arura, po pušó.

Bobobo, bobobo,

Siehe, ich bin gekommen, sei nun still.

Anm. $b o$ ist interjectionell. Es kommt in einem : alnnlichen liede in Reinholds Anthologie s. 20, 12 vor: haide ho, e bo bo bo!

2.

Nisciurura na civin

Ha pen derini ti hin.

I come I run; open the door that I may enter. na tš arura, na tš́ vin; hape dérens ts hin.

Siehe ich bin gekommen, siehe ich komme;

öffne die Thur, dass ich hineingehe.

A nm. Der 'unbestimmte vokal,' den ich mit $\varepsilon$ bezeichne, wird von Byron oder seinew gewiihrsmanne als $i$ geschrieben. Das $n$ von ha pen

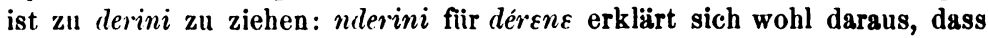
der schreiber, der native of Athens, ein Grieche war, der gewohnt war, die seiner sprache fehlende anlautende media $d$ durch $\nu \tau$ zu schreiben. Ebenso ist aufzufassen in 3 uderi, Druckfehler fuir nderi; 8 udo gia = ndogia $; 17$ udi $=$ ndi, udorini $=$ ndorini, udiri $=$ ndivi. 
3.

Ha pe uderi escrotini

$T i$ vin ti mar servetini.

Open the door by halves, that

I may take my turban.

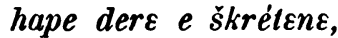

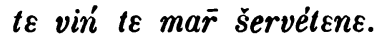

Oeffne die thur, die ungluckselige, dass ich komme, mein kopftuch zu nehmen.

A n m. by halves ist sicher ein misverständniss. ̌̀ervet $\varepsilon$, lehnwort aus trz. serviette, ist 'tuch,' das ja auch als turban verwendet werden kann, wie bei den ärmeren volksklassen der Türkei.

4.

Caliriote me surme

Ea ha pe pse duc tive.

Caliriotes with the dark eyes, open the gate, that I may enter.
Kaliriote me surmé, ea, hup, pse lua te vēe.

Mädchen mit den geschwärzten augenwimpern, komm, öffne, denn ich will gehen.

A n m. Was ich ïber Caliriote in meinem Etymologischen Würterbuche der alb. Sprache s. $167 \mathrm{f}$. gesagt habe, scheint mir heute nicht mehr sicher. Man kann dem worte vielleicht gerecht werden, ohne die gewaltsame änderung in Kuluriote vorzunehmen. Die alte beriihmte quelle, $K \alpha \lambda \lambda \iota \rho \varrho o ́ \eta$, noch heute Kalirói genannt, siidlich vom Olympieion, ist häufiges stelldichein von frauen und mädchen, die dort wasser holen oder wïsche waschen. Nach dem zeugnisse von Hobhouse (vgl. unten) 'Iravels II, 434 führten damals (1809/10) griechische framen in der wihe der quelle Kalirói eine bestimmte art tïnze aus. Also ist Caliviote wol 'Miidchen von Kalirúi.'

5.

Buo, Bo, Bo, Bo, Bo,

bo bo bo, bo bo bo,

Gi egem spirta esimiro.

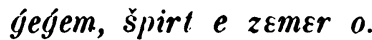

Lo, Lo, I hear thee, my soul.

bo bo bo, bo bo bo,

ich höre, o. (meine) seele und

(mein) herz.

6.

Caliriote vu le funde

Ede vele tunde tunde.

An Arnaout girl. in costly garlb, walks with graceful pride.
Kaliriote, vure funde ejé vete tunde-tunde.

Mädchen, du hast quasten angelegt und gehst schaukelnd daher. 
7.

Caliriole me surme

Ti mi put e poi mi le.

Caliriot maid of the dark eyes, give me a kiss.
Kaliriote me surmé,

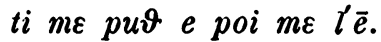

Mädchen mit den geschwärzten augenwimpern,

du kusst mich und dann verlässt du mich.

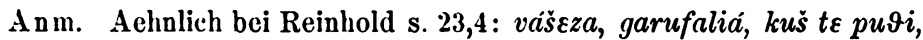
pra $t \varepsilon$ l'a? ' $O$ mädchen, du nelke, wer hat dich gekuisst und dann verlassen ?' Byron's Grieche hat die zweite zeile gar nicht verstanden.

8.

Se ti pula citi mora

Si mi ri ni veti udo gia.

If I have kissed thee, what hast thou gained? My soul is consumed with fire. se $t \varepsilon p u \vartheta a$, $t s ̌ \varepsilon ~ t \varepsilon$ mora?

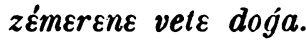

Wenn ich dich kủsste, was habe ich dir genommen? (mein) herz allein habe ich verbrannt.

9.

Va le ni il che cadale Celo more, more celo. Dance lightly, more gently, and gently still.

Anm. Dr. Nerutsos fasst $t t^{\prime} e l^{\prime} o$ als eigenname. Das ist wol nicht richtig. tisel' bedeutet 'zugïnglich, mann von welt' (Etym. Wörterbuch s. 446), und dazu stimmt Byrons gently. moré wird bei der anrede an männer gebraucht.

10.

\section{Plu hari li lirele}

Plu huron cia pra seti.

Make not so much dust to destroy your embroidered hose.

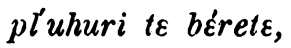
pl'uhurón tšaprizete.

Der staub wird dir gemacht (aufgewirbelt);

du bestaubst (deine) schntirenbesätze.

An m. Die erste zeile habe ich mit möglichstem anschlusse an Byron's text emendiert. 'Ich werde gemacht' heisst allerdings sonst benem oder behem; doch ist ein berem nach dem participium bere denkbar. Oder

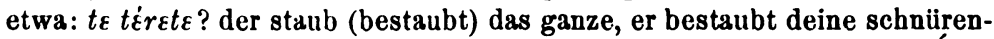
besätze. Dr. Nerutsos will herstellen: pl'uhurove tirkite (besser tirḱete) 'du hast deine gamaschen bestaubt,' was mir mit rücksicht auf die tiberlieferung und iibersetzung bei Byron etwas gewagt vorkommt. 
11.

Ndi sefda tinde ulavossa

Vettimi upri vi lofsa.

I am wounded by thy love, and have loved but to scorch myself. nde sevdá tende u-l'avosa,

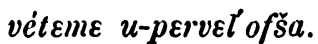

In der liebe $z u$ dir habe ich mich verwundet;

mich selbst habe ich versengt. 12.

Ah vaisisso mi privi lofse

Si mi rini mi la vosse.

Thou hast consumed me! Ab, maid! thou hast struck me to the heart.

\section{3.}

Uti tasa roba stua

Sitli eue tulali dua.

I have said I wish no dowry, but thine eyes and eye lashes.

14.

Roba stinori ssidua.

Qu mi sini vetti dua.

The accursed dowry I want not, but thee only.

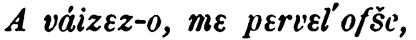
zémerene me ĺavose.

0 mädchen, du hast mich versengt,

das herz hast du mir verwundet.

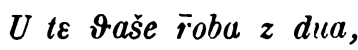

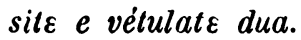

Ich sagte dir, kleider will ich nicht,

deine angen und augenbrauen will ich.

ioba stin orji $s$ i dua,

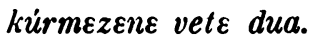

Kleider - sie seien verwunscht - will ich nicht;

deinen körper allein will ich.

Anm. stinori ist ngr. ' $\sigma \tau \grave{\eta} \nu$ óg $\eta^{\prime} . \quad s=$ 'nicht,' in $13 z$ vor der Media. $i$ ist anaphorisches Pronomen der 3. plural accusativ, auf $\bar{r} o b a$ bezliglich.

Qurmini dua civilem

Roba ti siarmi tildi eni.

Give me thy charms, and let the portion feed the flames.
15.

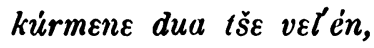
$\overline{r o ́ b a t \varepsilon ~ z i a r m i ~} t$ ' $i$ djekric.

Deinen körper will ich, der viel wert ist;

die kleider soll das fener ver. brennen.

A $\mathbf{n m}$. Die strophe ist nicht in ordnung. Die verbesserung ťs $v \varepsilon l^{\prime} e ́ n$ rührt von Dr. Nerutsos her. Aber es kann nicht richtig sein, dass die erste zeile männlich, die zweite weiblich ausgeht. Dus $l$ in tildi ist ein merkwürdiger zusatz, der auch in 16 in tiltati, 17 in cilti und talti wiederkehrt. 
16.

Ctara pisa vaisisso me simi rin ti hapti

Eti mi bire a piste si gui dendroi tillati.

I have loved thee, maid, with a sincere soul, but thou hast left me like a withered tree.

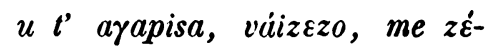
meren $t \varepsilon$ hapte,

$e$ ti $m \varepsilon$ bere, ápiste, si ri

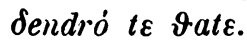

Ich habe dich geliebt, mädchen, mit aufrichtigem (eigentlich 'offenem') herzen, und du hast mich gemacht, treulose, zu (eig. 'wie') einem trockenen baume.

A $\mathbf{n}$ m. $r$ in utara pisa erkliart sich leicht aus der aussprache des

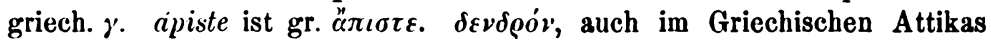
so betout, ist besonders der Oelbaum. gui druckfehler für gni.

\section{7.}

Udi vura udorini udiri cicova cilli mora

Udorini talli hollna $u$ ede caimoni mora.

If I have placed ny hand on thy bosom, what have I gained? my hand is withdrawn, but retains the flame. nde vura dórene ndere tsitsate, Iš $t \varepsilon$ mora?

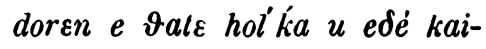
mone mora.

Wenn ich meine hand zwischen deine brtiste gelegt habe, was habe ich dir genommen? Die hand habe ich verdorrt (versengt) herausgezogen und verbrennung habe ich davon getragen.

A n m. tsitsate weicht von cicova stark ab, aber ich weiss sonst keinen rat. kaimone acc. von kaimó $=$ ngr. $x \alpha \ddot{\mu} \mu o ́ s$.

Das griechische original dieser strophe finde ich in einem volksliede,

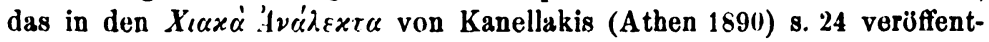
licht worden ist:

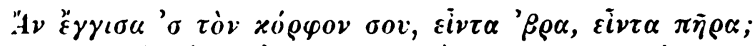

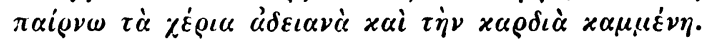

Auch der reisebegleiter Byron's auf seiner orientreise im jahre 1809, John Hob hou se, später Lord Broughto n, dem der vierte gesang des Childe Harold gewidmet ist, hat einige albanische fragmente in Attika aufgezeichnet, die er in seinem reisewerke mitteilt, in wesentlich schlechterer, stark anglisierender orthographie (Travels in Albania and other provinces of Turkey in 1809 and 1810. By the Right Hon. Lord Broughton. A new edition. London 1858. Vol. II, p. 433). 
Ich teile hier anhangsweise ihre verbesserte gestalt mit:

1.

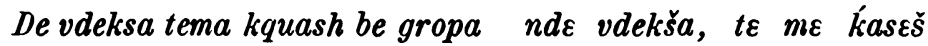
targalissa mbe gropa $t$ ' argal' ise,

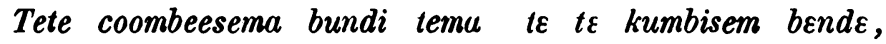
pustrosh me sissa.

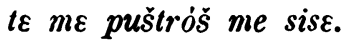

If $I$ die a virgin, bury me under your couch.

Wenn ich sterbe, nimm mich auf in das grab deines web-

When you go to your repose, I shall rest beneath your bosom. stuhles, damit ich dir zur seite lehne, damit du mich mit deinem busen bedeckst.

2.

$V$ dekea athe me savanosna

A pastai me metasnosa.

I am dead, and they have wrapped me in my windingsheet;

Now it is they sorrow for my death.

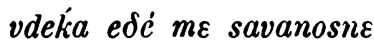

$c$ pastáj me metanosne.

Ich starb, und sie haben mich in das sterbekleid gewickelt, und dann haben sie fur mich gebetet.

3.

Vdekea athe de klish me kialn A pastai roune te kliagn.

I am dead, and they have borne me to the churchyard; there they have begun their lamentation.

Graz, im Februar 1892.

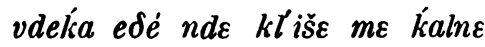

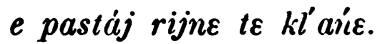
Ich starb, und sie brachten mich in die kirche, und dann setzten sie sich um za weinen.

Gustav Meyer. 